\title{
Three Important Refinements of the Condensed Matter Detonation Theory
}

\author{
V.S. Trofimov, E.V. Petrov* \\ Merzhanov Institute of Structural Microkinetics and Materials Science of RAS, \\ 8, Academician Osipyan Str., Chernogolovka, 432142, Russia \\ *Corresponding author. Tel.: +7 4965246 222.E-mail: pnkv@list.ru
}

\begin{abstract}
Some sections of the theory of shock-wave processes in condensed media require substantial refinement. From a detailed analysis of the concept of heat of reaction, it follows that the three known heats of reaction characterize the same transformation of the reacting medium from three different sides. When considering the flows of a reacting medium, it is necessary to take into account turbulence and its changes as an analogue of thermal motion and physicochemical transformations of matter. Based on the analysis of the results of a unique experiment, it was concluded that the deformation of the condensed medium is a factor that determines the reaction rate along with temperature.
\end{abstract}

\section{Keywords}

Explosive; heat of reaction; condensed medium; turbulence; reaction rate.

(C) V.S. Trofimov, E.V. Petrov, 2020

\section{Introduction}

This article analyses three fundamental issues to which insufficient attention is paid in the studies on the physics of explosion. The first question is that there are at least three different heats of the same reaction. But this is usually not taken into account in the studies on the physics of explosion. The second question - in the case of condensed media, turbulence is usually neglected, although it is turbulence, if present, that determines the structure of the shock or detonation wave. The third question is that temperature is usually considered the main external factor that determines the reaction rate. However, there is an experiment according to which the current deformation rate of the medium is also such a factor.

We consider these issues in more detail.

\section{Determination of the heat of reaction}

Researchers of explosives tend to believe that each explosive can be assigned a certain heat of reaction $Q(\alpha)$, where the chemical composition $\alpha$, for simplicity, will be considered one-component. Therefore, in studies of explosives, expressions of the form:

$$
E(X, Y, \alpha)=E_{h}(X, Y, \alpha)+Q(\alpha)
$$

are frequent, where $E$ is specific internal energy, $E_{h}$ is the measure of the thermal motion and mechanical stresses of the medium, here and in what follows arguments $X, Y$ represent a pair of independent parameters $(P, V),(P, T)$ or $(V, T)$. Here $P$ is pressure, $V$ is specific volume, $T$ is temperature.

However, for an unambiguous division of the internal energy of the medium into two parts, appropriate physical criteria are needed. In particular, a criterion is needed that allows dividing unambiguously the measure of motion of all valence electrons between mechanical stresses and chemical bonds. Since such criteria are not known today, it is better to abandon the division of the internal energy of the medium into parts as an unnecessary complication of the theory of explosives. Therefore, we will consider the internal energy of the reacting medium as a whole. This is considered in manuals on thermodynamics, in particular, in the well-known course of theoretical physics by L.D. Landau and E.M. Lifshitz $[1,2]$.

It follows from what has been said that the heat of reaction can be determined only in relation to a preselected initial state of the explosive. We denote the parameters of such a state by the index zero $-E_{0}, P_{0}$, $V_{0}, T_{0}, \alpha_{0}$ and represent it by the point $P_{0}, V_{0}$ on the $P-V$ plane (Fig. 1). 


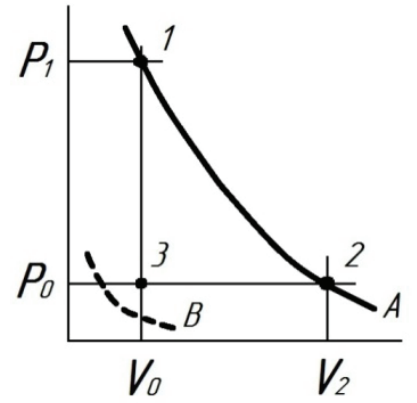

Fig. 1. Isotherms of reaction products on the $\boldsymbol{P}-\boldsymbol{V}$ plane: $A$ a for $T=T_{0} ; B$ for $T=0 \mathrm{~K}$

We consider the same chemical transformation of explosive $\alpha_{0} \rightarrow \alpha$, occurring under three different conditions - at constant $V$ and $T$, at constant $P$ and $T$, and at constant $P$ and $V$. The final composition is not necessarily equilibrium. The corresponding final states of the reaction products are designated on the $P-V$ plane by points 1,2 , and 3 (Fig. 1). Points 1 and 2 are located on the $T_{0}$ isotherm of the reaction products, and point 3 coincides with point $P_{0}, V_{0}$. It should be borne in mind that the state $P_{0}, V_{0}, \alpha$ exists for the reaction products only under the condition that the zero isotherm of these products $(T=0 \mathrm{~K})$ passes below the initial state of the explosive in pressure (Fig. 1).

We present the expressions of three heats of the same reaction $\alpha_{0} \rightarrow \alpha$ :

$$
\begin{aligned}
Q_{V} & =Q_{V T}=E\left(P_{0}, V_{0}, \alpha_{0}\right)-E\left(P_{1}, V_{0}, \alpha\right)= \\
& =E\left(V_{0}, T_{0}, \alpha_{0}\right)-E\left(P_{0}, V_{0}, \alpha\right), \\
Q_{P}= & Q_{P T}=E_{0}-E\left(P_{0}, V_{2}, \alpha\right)-P_{0}\left(V_{2}-V_{0}\right)= \\
& =E_{0}-E\left(P_{0}, T_{0}, \alpha\right)-P_{0}\left(V_{2}-V_{0}\right), \\
Q_{P V} & =E\left(P_{0}, V_{0}, \alpha_{0}\right)-E\left(P_{0}, V_{0}, \alpha\right) .
\end{aligned}
$$

Below, when using expression (3), it will be assumed that the state $P_{0}, V_{0}, \alpha$ for the reaction products exists.

As is known from [3], one more heat of reaction is determined in calorimetric bombs - the calorimetric heat of reaction $Q_{\text {cal }}$. An analysis of the processes in such bombs leads to the determination of the heat $Q_{\mathrm{cal}}$ and its relationship with $Q_{P T}$ :

$$
\begin{aligned}
Q_{\mathrm{cal}} & =E\left(P_{0}, V_{0}, \alpha_{0}\right)-E\left(P_{0}, V_{2}, \alpha\right)= \\
& =E\left(P_{0}, T_{0}, \alpha_{0}\right)-E\left(P_{0}, T_{0}, \alpha\right), \\
Q_{P T} & =Q_{\mathrm{cal}}-P_{0}\left(V_{2}-V_{0}\right) .
\end{aligned}
$$

As you know, the last expression is used to calculate $Q_{P}$ for $Q_{\text {cal }}$.
In many studies, the heat $Q_{\text {cal }}$ (4) is often denoted as $Q_{V}$ and is identified with the heat $Q_{V T}$ (1), and expression (5) is interpreted as the ratio between $Q_{P}$ and $Q_{V}$ (see in [3, p. 126]).

This is an error, and indeed, according to equations (1) and (4), from $Q_{\mathrm{cal}}=Q_{V T}$ it follows $E\left(V_{0}, T_{0}, \alpha\right)=E\left(P_{0}, T_{0}, \alpha\right)$. The last equality is possible if only on the entire isotherm $T_{0}$ between points 1 and 2 (Fig. 1) the products satisfy the Mendeleev-Clapeyron equation. But this is certainly not true at point 1 , where the products are compressed to the density of the condensed explosive.

We define the role of each heat of reaction in physical processes.

We start by considering the heat $Q_{P V}$, proposed in monograph [4]. From the expression for the energy conservation law in a shock jump $[2,4]$ :

$$
E(P, V, \alpha)-E\left(P_{0}, V_{0}, \alpha_{0}\right)=\frac{\left(P+P_{0}\right)\left(V-V_{0}\right)}{2}
$$

taking into account $Q_{P V}(3)$ we obtain:

$$
E(P, V, \alpha)-E\left(P_{0}, V_{0}, \alpha\right)=\frac{\left(P+P_{0}\right)\left(V_{0}-V\right)}{2}+Q_{P V} .
$$

It is easy to show that expression (6) if $Q_{P V}>0$ is detonation adiabat, and if $Q_{P V}=0$ it is a shock adiabat without reaction, and if $Q_{P V}<0$ it is shock adiabat with reaction. Thus, the heat of reaction $Q_{P V}$ (3) determines the type of shock-wave process.

To determine the role of different heats of reaction in a shockless flow, we turn to the first law of thermodynamics:

$$
\frac{d E}{d t}=\frac{d q}{d t}-P \frac{d V}{d t},
$$

where $t$ is time, $q$ is heat entering the considered particle of reaction products from the outside, i.e. from neighboring particles.

We differentiate in expression (7) $E$ and $V$ as complex functions of $t$ taking into account the determination of the heats of reaction (1) - (3) and thermodynamic identities:

$$
\left(\left(\frac{\partial E}{\partial X}\right)_{Y \alpha}+P\left(\frac{\partial V}{\partial X}\right)_{Y \alpha}\right)\left(\frac{\partial X}{\partial Y}\right)_{S \alpha}=-\left(\frac{\partial E}{\partial Y}\right)_{X \alpha}-P\left(\frac{\partial V}{\partial Y}\right)_{X \alpha},
$$

where $S$ is specific entropy, while $X$ and $Y$ represent $V$ and $P, V$ and $T$ or $P$ and $V$. The obtained expressions are transformed using the formulas for the specific heat capacities $C_{P}, C_{V}$ and the Grüneisen coefficient $\Gamma$ : 


$$
\begin{gathered}
C_{P}=\left(\frac{\partial E}{\partial T}\right)_{P \alpha}+P\left(\frac{\partial V}{\partial T}\right)_{P \alpha} ; \\
C_{V}=\left(\frac{\partial E}{\partial T}\right)_{V \alpha} ; \quad \Gamma=V\left(\frac{\partial P}{\partial E}\right)_{V, \alpha} .
\end{gathered}
$$

These formulas are actually definitions of $C_{P}, C_{V}$ and $\Gamma$.

As a result, we find:

$$
\begin{aligned}
& \frac{\partial T}{\partial t}=\left(\frac{\partial T}{\partial V}\right)_{S \alpha} \frac{d V}{d t}+\frac{1}{C_{V}}\left(\frac{d Q_{V T}}{d t}+\frac{d q}{d t}\right) ; \\
& \frac{\partial T}{\partial t}=\left(\frac{\partial T}{\partial P}\right)_{S \alpha} \frac{d P}{d t}+\frac{1}{C_{P}}\left(\frac{d Q_{P T}}{d t}+\frac{d q}{d t}\right) ; \\
& \frac{\partial P}{\partial t}=\left(\frac{\partial P}{\partial V}\right)_{S \alpha} \frac{d V}{d t}+\frac{\Gamma}{V}\left(\frac{d Q_{P V}}{d t}+\frac{d q}{d t}\right) .
\end{aligned}
$$

Note that $q$ and $Q_{X Y}$ enter each of expressions $(10)-(12)$ in the same way, i.e. each heat of reaction in the equations plays the same role as the external heat.

From expressions (6), (10) - (12) it follows that of the three heats of reaction $Q_{V T}, Q_{P T}$ and $Q_{P V}$, the main one cannot be distinguished. Each of them plays a decisive role in the movements of the environment. This must be borne in mind when analyzing and calculating shock-wave processes.

\section{On turbulent shock-wave motion}

In condensed reacting media, a turbulent shockwave process is often observed. It arises due to the initial inhomogeneity of the medium and the instability of its laminar motion. In the theories of such a process, equations are developed for the averaged motion of a plane turbulent stationary shock or detonation wave in a charge of physically infinitely large diameter in the frame of reference associated with this wave (Fig. 2).

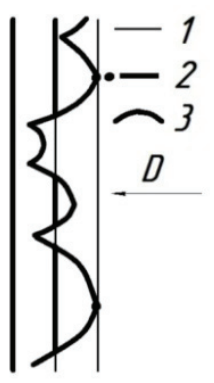

Fig. 2. The structure of a shock jump in a turbulent shock-wave process: 1 - section part free of the $\delta$-section; $2-\delta$-section; 3 - shock front
We consider the most developed of these theories $[4,5]$. Its peculiarity lies in the fact that the parameters of the medium flow are averaged not only over the cross sections of the charge, but also over the parts of these sections, which are called $\delta$-sections (Fig. 2). The $\delta$-section is determined as a part of the section, to which the initial flow of the medium does not reach. At each section, the $\delta$-section changes its configuration over time, but keeps its area constant due to the stationarity of the process. This area, with distance from the beginning of the wave, increases from zero at the beginning of the wave to the cross-sectional area.

Another feature of the theory $[4,5]$ is that only a part of the parameters, among which the density $\rho$, is averaged with a weight function equal to unity. The corresponding averages are denoted by the bar above. The other part of the parameters is averaged with the weight function $\rho / \bar{\rho}$, and the corresponding averages are denoted by two bars above.

When averaging over the sections and over the $\delta$-sections, we obtain the same type of system of shockwave equations:

$$
\begin{gathered}
\rho_{0} D=\bar{\rho}(D-\overline{\bar{u}}) ; \\
P_{e f}-P_{0}=\rho_{0} D \bar{u} ; \\
E_{e f}-E_{0}=\frac{\left(P_{e f}+P_{0}\right)\left(V_{0}-\overline{\bar{V}}\right)}{2}+\frac{q_{e f}}{\rho_{0} D} .
\end{gathered}
$$

Here $D$ and $u$ are the wave and mass velocities of the shock or detonation wave, respectively, effective pressure $P_{e f}$, effective internal energy $E_{e f}$ and effective heat flux $q_{e f}$ are determined by the expressions:

$$
\begin{aligned}
& P_{e f}=\bar{P}+\overline{\rho u_{x}^{2}} ; \\
& E_{e f}=\overline{\bar{E}}+\frac{\overline{u_{x}^{2}+u_{y}^{2}+u_{z}^{2}}}{2} ; \\
& q_{e f}=\overline{P u_{x}}+\rho u_{x}\left(E+\frac{u_{x}^{2}+u_{y}^{2}+u_{z}^{2}}{2}\right) .
\end{aligned}
$$

where $u_{x}, u_{y}, u_{z}$ are turbulent pulsation velocity components. In the case of isotropic turbulence, $q_{e f}=0$. In a turbulent shock-wave process, the effective quantities $P_{e f}, E_{e f}$ and $q_{e f}$ behave similarly to the corresponding quantities in a laminar process.

In the system of equations (13) - (18), obtained by averaging over the cross sections, the initial point of the shock or detonation wave lies at the pressure $P_{0}$. On the contrary, in the system of equations (13) - (18), 
obtained by averaging over $\delta$-sections, the initial point of the shock or detonation wave lies at the peak of the first shock jump. This makes it possible to apply to turbulent detonation the justification for the selection rule for the velocity $D$ taken from the theory of laminar detonation by Ya.B. Zeldovich [1].

Attenuation of turbulence in the flow of the medium manifests itself as a chemical reaction. This is from expressions (16), (17). For this reaction, the sign of the heat $Q_{P V}$ (3) depends on the Grüneisen coefficient of the medium $\Gamma$ (9). If $\Gamma>2 / 3 Q_{P V}>0$, but if $\Gamma<2 / 3 Q_{P V}<0$. Therefore, when this reaction is complete in the detonation wave, the detonation will be normal if the medium has $\Gamma>2 / 3$, but it is incomplete, if $\Gamma<2 / 3$.

According to expressions (13) - (18), within the limits from the beginning of the shock or detonation wave to the point where the $\delta$-section occupies the entire section, turbulence cannot be neglected. Here, it is the main factor that determines the internal structure of the shock wave or detonation wave.

\section{On the dependence of the reaction rate on the deformation rate}

We consider the unusual dependences $D(L)$ shown in Fig. 3. They were obtained in experiments performed in $[6,7]$ on the initiation of a shock wave with a plane front, and a conclusion was drawn about the mechanism of excitation of detonation by a shock wave in cast TNT. Under the action of the shock wave, detonation occurs almost instantly at the interface in an imperfect mode. The amplitude of the initiating shock wave is insufficient to induce a chemical reaction similar to the chemical reaction in ideal detonation. Here $D$ and $L$ are the shock jump velocity and its path in the TNT charge, respectively. Curve $l$ was obtained in [6], curve $2-$ in [7]. Of fundamental importance is the fact that on both $D(L)$ dependences there are sections of constant velocity - "induction periods".

Assuming the Arrhenius dependence of the reaction rate on temperature, it is possible to approximately reproduce in calculations the shape of each of these curves separately by choosing the preexponent and activation energy. However, both of these curves cannot be reproduced at once using the same dependence of the reaction rate on temperature.

Hence it follows that not the temperature, but another factor determines the reaction rate under the conditions of the observed shock-wave process.

From a comparison of curves 1 and 2 (Fig. 3) it follows that such a factor can only be the rate of deformation of the reacting medium. The same conclusion was made earlier in [8] based on an analysis of only one curve 2 and the associated oscillograms of the mass velocity taken from [6].

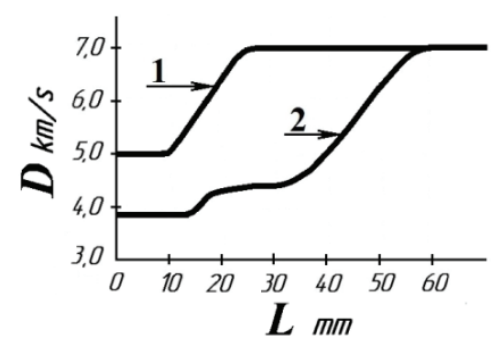

Fig. 3. Development of detonation upon initiation of cast TNT by shock waves of a rectangular profile

The possible dependence of the reaction rate on the deformation rate of the reacting medium must be borne in mind when analyzing and calculating shockwave processes in condensed media.

\section{Conclusion}

The performed analysis showed that, at least in the case of shock-wave processes in condensed media, there is no such unified characteristic of the chemical transformation of a substance as the heat of reaction. In each specific case, you need to know which of these characteristics should be taken into account.

In the case of condensed media, turbulence cannot be neglected. It plays a role as an additional component of the chemical composition of the medium and as an additional factor in the thermal motion of the medium.

When analyzing shock-wave processes in condensed media, it should be borne in mind that the rate of a chemical reaction can be determined not only by the temperature of the reacting medium, but also by its current deformation.

\section{References}

1. Landau L.D., Lifshits E.M. Teoreticheskaya fizika: Statisticheskaya fizika [Theoretical Physics: Statistical Physics], Moscow, Fizmatlit Publ., 2002. 616 p. (Rus).

2. Landau L.D., Lifshits E.M. Teoreticheskaya fizika: Gidrodinamika [Theoretical physics: Hydrodynamics], Moscow, Fizmatlit Publ., 2001. 736 p. (Rus).

3. Andreev S.G., Babkin A.V., Baum F.A. et al. Fizika vzryva [Explosion physics], Moscow, Fizmatlit Publ., 2002. 823 p. (Rus).

4. Dremin A.N., Savrov S.D., Trofimov V.S., Shvedov V.S. Detonatsionnye volny v kondensirovannykh sredakh [Detonation waves in condensed media], Moscow, Nauka Publ., 1970. 162 p. (Rus).

5. Trofimov V.S. Turbulent detonation theory. Doklady Physical Chemistry, 2009, 428(2), 777-779. doi: 10.1134/S0012501609100078.

6. Dremin A.N., Koldunov S.A., Shvedov K.K. Initsiirovanie detonatsii udarnoy volnoy $\mathrm{v}$ litom trotile [Detonation initiation by a shock wave in cast TNT], Prikladnaya mekhanika i tekhnicheskaya fizika, 1963, 6, 131-134. (Rus).

7. Dremin A.N., Koldunov A.S. Initsiirovanie detonatsii udarnymi volnami $\mathrm{v}$ litom i pressovannom trotile [Initiation of detonation by shock waves in cast and pressed TNT], Sbornik Vzryvnoe delo, 1967, 63/20, 37-50. (Rus).

8. Trofimov V.S., Trofimova G.P. Simple proof of the dependence of the reaction rate on the rate of substance deformation in a detonation wave (DW). Combustion, Explosion and Shock Waves, 1990, 26(1), 121-127. doi: 10.1007/BF00742287 